\title{
Synthesis and characterization of fully substituted pyrimidines by using ketene dithioacetal as potent antimicrobial agent
}

\author{
Hasmukh R. Khunt, Piyush P. Pipaliya, Satish M. Ghelani, Jayesh S. Babariya, \\ Yogesh T. Naliapara* \\ Department of Chemistry, Saurashtra University, Rajkot, Gujarat, India \\ *E-mail address: naliaparachem@yahoo.co.in
}

\begin{abstract}
Various ketene dithioacetals of acetoacetanilides were reacted with guanidine nitrate in the presence of base to produce the 2-amino-4-isopropyl-6-alkoxy- $N$-arylpyrimidine-5-carboxamide derivatives with good yields. All the synthesized compounds were characterized by mass, NMR and IR and also evaluated for antimicrobial activity against five different bacterial and fungal strains. The compounds $4 \mathrm{i}, 4 \mathrm{k}$ and $4 \mathrm{l}$ has found comparatively good active against all the bacterial strains.
\end{abstract}

Keywords: Ketene dithioacetal; Pyrimidines; Guanidine; Biginelli reaction

\section{INTRODUCTION}

From the beginning of pharmaceutical research nitrogen containing heterocyclic compounds have been considered as the privileged structure because of the different biological property and its tremendous applications e.g., anticancer, diuretic, anticonvulsant, anti-inflammatory and antihypertensive activities [1]. Particularly pyridine nucleus which is the important constructive part of many natural products and used as antimalarial [2], antitoxoplasma [3], fungicidal [4], antibacterial [5], anti-inflammatory and antitumor agent [6]. The Biginelli reaction [7] has been reported for the synthesis of many pyrimidine darivatives by the one-pot condensation of active methylene, urea and aldehydes. There are some other methods are also reported with some little modification in Biginelli reaction by the condensation of aldehydes, urea and arylketones in acetic acid using a catalytic amount of KHSO4 [8], in basic condition [9] and by microwave irradiation [10]. Now a days of literature have been reported for the synthesis of thiophene, furan, isooxazole, pyrazole, pyridine and pyrimidine etc. heterocycles by using dithioacetal in place of aldehyde $[11,12]$. Thus, above literature promote us to synthesize novel pyrimidine analogues using ketene dithioacetal and their biological screening. 


\section{RESULT AND DISCUSSION}

In our current research variety of acetoacetanilides 2a-t were prepared from methyl 4methyl-3-oxopentanoate (Scheme 1). These acetoacetanilides on reaction with $\mathrm{CS}_{2}$ and methyl iodide in presence of potassium carbonate produce ketene dithioacetal 3a-t (Scheme 2) on active methylene. These dithioacetals are behave as a precursor of aldehyde, which on reaction with guanidine nitrate in presence of sodium methoxide or sodium ethoxide lead to the formation of fully substituted pyrimidine 4a-t (Scheme 2). Here base replaces one thiomethoxy group and after cyclization methoxy or ethoxy group is introdused at the position of thiomethoxy group. Varoius ketenedithioacetal was used for the synthesis of pyrimidine, is listed in Table 1 .<smiles>[R]NC(=O)CC(=O)C(C)C</smiles>

Scheme 1. Stnthesis of acetoacetanilide.<smiles>[R]NC(=O)CC(=O)C(C)C</smiles>

Scheme 2. Synthesis of pyrimidine via ketenedithioacetal.

Table 1. Various ketene dithioacetal used for the synthesis of pyrimidines.

\begin{tabular}{cccccc}
\hline Entry & Code & $\mathbf{R}$ & $\mathbf{R}_{\mathbf{1}}$ & $\begin{array}{c}\text { Yield } \\
\text { (\%) }\end{array}$ & $\begin{array}{c}\text { Time } \\
\text { (h) }\end{array}$ \\
\hline 1 & $4 \mathrm{a}$ & $4-\mathrm{BrC}_{6} \mathrm{H}_{4}$ & $\mathrm{C}_{2} \mathrm{H}_{5}$ & 92 & 6.0 \\
\hline 2 & $4 \mathrm{~b}$ & $4-\mathrm{OCH}_{3} \mathrm{C}_{6} \mathrm{H}_{4}$ & $\mathrm{CH}_{3}$ & 91 & 6.0 \\
\hline 3 & $4 \mathrm{c}$ & $\mathrm{C}_{6} \mathrm{H}_{5}$ & $\mathrm{CH}_{3}$ & 84 & 5.7 \\
\hline 4 & $4 \mathrm{~d}$ & $\mathrm{C}_{6} \mathrm{H}_{11}$ & $\mathrm{C}_{2} \mathrm{H}_{5}$ & 90 & 5.0 \\
\hline 5 & $4 \mathrm{e}$ & $2,5-\mathrm{di}^{-} \mathrm{CH}_{3} \mathrm{C}_{6} \mathrm{H}_{3}$ & $\mathrm{CH}_{3}$ & 86 & 5.5 \\
\hline 6 & $4 \mathrm{f}$ & $\mathrm{C}_{6} \mathrm{H}_{11}$ & $\mathrm{CH}_{3}$ & 92 & 5.6 \\
\hline 7 & $4 \mathrm{~g}$ & $4-\mathrm{FC}_{6} \mathrm{H}_{4}$ & $\mathrm{CH}_{3}$ & 90 & 6.0 \\
\hline 8 & $4 \mathrm{~h}$ & $4-\mathrm{ClC}_{6} \mathrm{H}_{4}$ & $\mathrm{C}_{2} \mathrm{H}_{5}$ & 86 & 5.8 \\
\hline
\end{tabular}




\begin{tabular}{cccccc}
\hline 9 & $4 \mathrm{i}$ & $3-\mathrm{Cl}, 4-\mathrm{FC}_{6} \mathrm{H}_{3}$ & $\mathrm{CH}_{3}$ & 93 & 5.5 \\
\hline 10 & $4 \mathrm{j}$ & $3,4-\mathrm{di}^{-} \mathrm{FC}_{6} \mathrm{H}_{3}$ & $\mathrm{CH}_{3}$ & 91 & 6.0 \\
\hline 11 & $4 \mathrm{k}$ & $3-\mathrm{ClC}_{6} \mathrm{H}_{4}$ & $\mathrm{CH}_{3}$ & 88 & 5.4 \\
\hline 12 & 41 & $3,4-\mathrm{di}^{-} \mathrm{ClC}_{6} \mathrm{H}_{3}$ & $\mathrm{CH}_{3}$ & 92 & 5.7 \\
\hline 13 & $4 \mathrm{~m}$ & $4-\mathrm{CH}_{3} \mathrm{C}_{6} \mathrm{H}_{4}$ & $\mathrm{CH}_{3}$ & 90 & 5.6 \\
\hline 14 & $4 \mathrm{n}$ & $2-\mathrm{CH}_{3} \mathrm{C}_{6} \mathrm{H}_{4}$ & $\mathrm{CH}_{3}$ & 87 & 5.8 \\
\hline 15 & $4 \mathrm{o}$ & $2-\mathrm{OCH}_{3} \mathrm{C}_{6} \mathrm{H}_{4}$ & $\mathrm{CH}_{3}$ & 85 & 5.5 \\
\hline 16 & $4 \mathrm{p}$ & $2-\mathrm{FC}_{6} \mathrm{H}_{4}$ & $\mathrm{CH}_{3}$ & 93 & 6.0 \\
\hline 17 & $4 \mathrm{q}$ & $2-\mathrm{BrC}_{6} \mathrm{H}_{4}$ & $\mathrm{CH}_{3}$ & 90 & 5.6 \\
\hline 18 & $4 \mathrm{r}$ & $\mathrm{C}_{6} \mathrm{H}_{5}$ & $\mathrm{C}_{2} \mathrm{H}_{5}$ & 89 & 6.0 \\
\hline 19 & $4 \mathrm{~s}$ & $4-\mathrm{ClC}_{6} \mathrm{H}_{4}$ & $\mathrm{CH}_{3}$ & 85 & 5.7 \\
\hline 20 & $4 \mathrm{t}$ & $3-\mathrm{ClC}_{6} \mathrm{H}_{4}$ & $\mathrm{C}_{2} \mathrm{H}_{5}$ & 92 & 5.4 \\
\hline
\end{tabular}

\section{1. Experimental}

Thin-layer chromatography was accomplished on 0.2-mm precoated plates of silica gel G60 $\mathrm{F}_{254}$ (Merck). Visualization was made with UV light (254 and $\left.365 \mathrm{~nm}\right)$. IR spectra were recorded on a FT-IR-8400 spectrophotometer using DRS prob. ${ }^{1} \mathrm{H}(400 \mathrm{MHz})$ and ${ }^{13} \mathrm{C}(100$ $\mathrm{MHz}$ ) NMR spectra were recorded on a Bruker AVANCE II spectrometer in $\mathrm{CDCl}_{3}$. Chemical shifts are expressed in $\delta$ ppm downfield from TMS as an internal standard. Mass spectra were determined using direct inlet probe on a GCMS-QP 2010 mass spectrometer (Shimadzu). Melting points were measured in open capillaries and are uncorrected.

\section{1. 1. General procedure for the synthesis of substituted isopropylacetoacetanilide(2a-t)}

A mixture containing the primary amine $(10 \mathrm{mmol})$, methyl isobutyrylacetate $(10 \mathrm{mmol})$ and catalytic amount of sodium or potassium hydroxide lie $(10 \%)$ was reflux at $110^{\circ} \mathrm{C}$ for the approximately $15-20 \mathrm{~h}$. The reaction was monitored by TLC. After completion of reaction, the solvent was removed under vacuum. The solid or oil was crystallized from methanol to give pure product $2 \mathrm{a}-\mathrm{t}$.

\section{1. 2. General procedure for the synthesis of ketene dithioacetals (3a-t)}

A mixture of 4-methyl-3-oxo- $N$-arylpentanamide 2a-t, $(10 \mathrm{mmol})$ and dried $\mathrm{K}_{2} \mathrm{CO}_{3}(10$ mmol $)$ in DMF was stirred for $2 \mathrm{~h}$ at room temperature. Then $\mathrm{CS}_{2}(30 \mathrm{mmol})$ was added and the mixture was stirred for an additional $2 \mathrm{~h}$ at room temperature. Methyl iodide $(20 \mathrm{mmol})$ was added at $0-5{ }^{\circ} \mathrm{C}$ and reaction mixture was stirred again for $4 \mathrm{~h}$ at $\mathrm{RT}$. After completion of reaction, reaction mixture was poured in to cold water $(40 \mathrm{~mL})$. The precipitated crude product was filtered and crystallized in EtOH.

\section{1. 3. General procedure for the synthesis of 2 -amino-4,6-disubstituted pyrimidine-5- carboxamide derivatives (4a-t)}

Ketene dithioacetals $3 \mathrm{a}-\mathrm{t}(10 \mathrm{mmol})$ was added in the mixture of guanidine nitrate $(10$ mmol) and sodium methoxide or sodium ethoxide $(20 \mathrm{mmol})$ in methanol or ethanol within 10-15 min. The resulting reaction mixtures was further stirred at room temperature for $15 \mathrm{~min}$ then refluxed for $6 \mathrm{~h}$. After completion of the reaction, the mixture was poured in to cold water. Precipitated solid was filtered, wash with water, dried and crystallization from EtOH to afford analytically pure products $4 \mathrm{a}-\mathrm{t}$. 


\section{1. 4. Spectroscopic data for the compounds 4a-t}

2-Amino- $\mathrm{N}$-(4-bromophenyl)-4-ethoxy-6-isopropylpyrimidine-5-carboxamide(4a):

white solid, mp 185-187 C; IR (KBr): 3459, 3327, 3193, 2999, 1648, 1586, 1261, $1061 \mathrm{~cm}^{-1}$; ${ }^{1} \mathrm{H}$ NMR: $\delta 1.18-1.20\left(\mathrm{~d}, 6 \mathrm{H}, 2 \mathrm{x} \mathrm{i}_{\mathrm{iCH}}\right), 1.29-1.33\left(\mathrm{t}, 3 \mathrm{H}, \mathrm{CH}_{3}\right), 3.07-3.13(\mathrm{~m}, 1 \mathrm{H}, \mathrm{i} p \mathrm{prH})$, 4.32-4.37 (q, 2H, $\left.\mathrm{CH}_{2}\right), 5.86\left(\mathrm{~s}, 2 \mathrm{H}, \mathrm{NH}_{2}\right), 7.38-7.40(\mathrm{~d}, 2 \mathrm{H}, \mathrm{Ar}-\mathrm{H}, j=8.8 H z), 7.63-7.65(\mathrm{~d}$, $2 \mathrm{H}, \mathrm{Ar}-\mathrm{H}, \quad j=8.8 \mathrm{~Hz}), 9.97(\mathrm{br}, \mathrm{s}, 1 \mathrm{H},-\mathrm{CONH})$; $\mathrm{MS}(\mathrm{m} / \mathrm{z}): 379\left(\mathrm{M}^{+}\right)$; Anal. Calcd for $\mathrm{C}_{16} \mathrm{H}_{19} \mathrm{BrN}_{4} \mathrm{O}_{2}$ : C, 50.67; H, 5.05; N, 14.77; Found: C, 50.48; H, 5.15; N, 14.52 .

2-Amino-4-isopropyl-6-methoxy- $\boldsymbol{N}$-(4-methoxyphenyl)pyrimidine-5-carboxamide(4b):

Yellow solid; mp 180-184 ${ }^{\circ} \mathrm{C}$; IR (KBr): 3452, 3307, 3223, 2980, 1653, 1509, 1461, $1051 \mathrm{~cm}^{-}$ 1; ${ }^{1} \mathrm{H}$ NMR: $\delta 1.19-1.25$ (d, 6H, $\left.2 \times{ }^{\mathrm{i}} \mathrm{prCH}_{3}\right), 3.07-3.13$ (m, $\left.1 \mathrm{H},{ }_{\mathrm{i}} \mathrm{prCH},\right), 3.78$ (s, 3H, $\left.\mathrm{OCH}_{3}\right)$, $3.82\left(\mathrm{~s}, 3 \mathrm{H}, \mathrm{OCH}_{3}\right), 5.69\left(\mathrm{~s}, 2 \mathrm{H}, \mathrm{NH}_{2}\right), 6.82-6.85(\mathrm{~d}, 2 \mathrm{H}, \mathrm{Ar}-\mathrm{H}, j=8.8 \mathrm{~Hz}), 7.60-7.62(\mathrm{~d}, 2 \mathrm{H}$, Ar-H, $j=8.8 H z), 9.58(\mathrm{~s}, 1 \mathrm{H}, \mathrm{CONH}) ; \mathrm{MS}(\mathrm{m} / z): 316\left(\mathrm{M}^{+}\right)$; Anal. Calcd for $\mathrm{C}_{16} \mathrm{H}_{20} \mathrm{~N}_{4} \mathrm{O}_{3}$ : C, 60.75; H, 6.37; N, 17.71; Found: C, 60.48; H, 6.15; N, 17.52 .

\section{2-Amino-4-isopropyl-6-methoxy- $N$-phenylpyrimidine-5-carboxamide (4c):}

Yellow solid; mp 210-212 C; IR (KBr): 3412, 3317, 3253, 2950, 1613, 15039, 1431, 1041 $\mathrm{cm}^{-1}$; ${ }^{1} \mathrm{H}$ NMR: $\delta 1.19-1.25\left(\mathrm{~d}, 6 \mathrm{H}, 2 \mathrm{x}{ }_{\mathrm{i}} \mathrm{prCH}_{3}\right), 3.07-3.13\left(\mathrm{~m}, 1 \mathrm{H},{ }_{\mathrm{i}} \mathrm{prCH},\right), 3.82(\mathrm{~s}, 3 \mathrm{H}$, $\left.\mathrm{OCH}_{3}\right), 5.69\left(\mathrm{~s}, 2 \mathrm{H}, \mathrm{NH}_{2}\right), 9.58(\mathrm{~s}, 1 \mathrm{H}, \mathrm{CONH}), 6.60-6.62(\mathrm{~d}, 2 \mathrm{H}, \mathrm{Ar}-\mathrm{H}, j=8.8 \mathrm{~Hz}), 7.10-7.13$ (m, 2H, Ar-H), 6.40-6.43 (m, 1H, Ar-H); MS (m/z): $286\left(\mathrm{M}^{+}\right)$; Anal. Calcd for $\mathrm{C}_{15} \mathrm{H}_{18} \mathrm{~N}_{4} \mathrm{O}_{2}$ : C, 60.92; H, 6.34; N, 19.57; Found: C, 60.48; H, 6.15; N, 19.32 .

\section{2-Amino- $N$-cyclohexyl-4-ethoxy-6-isopropylpyrimidine-5-carboxamide (4d):}

white solid; mp 196-198 ${ }^{\circ}$; IR (KBr): 3429, 3307, 3123, 2959, 1658, 1546, 1265, $1041 \mathrm{~cm}^{-1}$; ${ }^{1} \mathrm{H}$ NMR: $\delta 1.19-1.20\left(\mathrm{~d}, 6 \mathrm{H}, 2 \times{ }^{\mathrm{i}} \mathrm{prCH}_{3}\right), 1.22-1.25\left(\mathrm{t}, 3 \mathrm{H}, \mathrm{CH}_{3}\right), 1.28-1.93(\mathrm{~m}, 10 \mathrm{H}, 5 \mathrm{x}$ $\left.\mathrm{CH}_{2}\right), 3.07-3.14(\mathrm{~m}, 1 \mathrm{H}, \mathrm{prCH}), 3.79-3.86(\mathrm{~m}, 1 \mathrm{H}, \mathrm{CH}), 4.26-4.31$ (q, $\left.2 \mathrm{H}, \mathrm{CH}_{2}\right), 5.73(\mathrm{~s}, 2 \mathrm{H}$, $\left.\mathrm{NH}_{2}\right), 7.21(\mathrm{~s}, 1 \mathrm{H}, \mathrm{CONH})$; $\mathrm{MS}(\mathrm{m} / z): 306\left(\mathrm{M}^{+}\right)$; Anal. Calcd for $\mathrm{C}_{16} \mathrm{H}_{26} \mathrm{~N}_{4} \mathrm{O}_{2}: \mathrm{C}, 62.72 ; \mathrm{H}$, 8.55; N, 18.29; Found: C, 62.38; H, 8.10; N, 18.

\section{2-Amino-4-isopropyl-6-methoxy- $N$-(2,5-dimethylphenyl)pyrimidine-5-carboxamide(4e):}

yellow solid; mp 186-188 C; IR (KBr): 3420, 3341, 32631, 2930, 1610, 1553, $1411,1060 \mathrm{~cm}^{-}$ ${ }^{1}$; ${ }^{1} \mathrm{H}$ NMR: $\delta$ 1.19-1.25 (d, 6H, $\left.2 \times{ }^{\mathrm{i}} \mathrm{prCH}_{3}\right), 3.07-3.13\left(\mathrm{~m}, 1 \mathrm{H},{ }_{\mathrm{i}} \mathrm{prCH},\right), 3.82\left(\mathrm{~s}, 3 \mathrm{H}, \mathrm{OCH}_{3}\right)$, $5.69\left(\mathrm{~s}, 2 \mathrm{H}, \mathrm{NH}_{2}\right), 9.58(\mathrm{~s}, 1 \mathrm{H}, \mathrm{CONH}), 6.62(\mathrm{~s}, 1 \mathrm{H}, \mathrm{Ar}-\mathrm{H}), 6.90-6.92,(\mathrm{~d}, 1 \mathrm{H}, \mathrm{Ar}-\mathrm{H}, j=8.8 \mathrm{~Hz})$, 6.72-6.74(d,1H, Ar-H, $j=8.8 H z), 2.34$, $\left(\mathrm{s}, 3 \mathrm{H}, \mathrm{CH}_{3}\right), 2.32,\left(\mathrm{~s}, 3 \mathrm{H}, \mathrm{CH}_{3}\right) ; \mathrm{MS}(\mathrm{m} / \mathrm{z}): 314\left(\mathrm{M}^{+}\right)$; Anal. Calcd for $\mathrm{C}_{17} \mathrm{H}_{22} \mathrm{~N}_{4} \mathrm{O}_{2}$ : C, 64.58; H, 7.05; N, 17.82; Found: C, 64.48; H, 7.15; N, 17.62.

\section{2-Amino- $N$-cyclohexyl-4-isopropyl-6-methoxypyrimidine-5-carboxamide(4f):}

yellow solid; mp 190-192 C; IR (KBr): 3462, 3307, 3223, 2990, 1653, 1509, 1461, $1061 \mathrm{~cm}^{-1}$; ${ }^{13} \mathrm{C}$ NMR: $\delta$ 21.20, 24.42, 25.02, 31.60, 32.16, 38.90-40.16, 47.97, 53.01, 106.73, 161.89, 164.94, 166.47, 173.41. ${ }^{1} \mathrm{H}$ NMR: $\delta 1.19-1.25\left(\mathrm{~d}, 6 \mathrm{H}, 2 \times \mathrm{i}^{\mathrm{i}} \mathrm{prCH}_{3}\right), 3.07-3.13\left(\mathrm{~m}, 1 \mathrm{H},{ }_{\mathrm{i}}^{\mathrm{i}} \mathrm{prCH},\right)$, $3.82\left(\mathrm{~s}, 3 \mathrm{H}, \mathrm{OCH}_{3}\right), 5.69\left(\mathrm{~s}, 2 \mathrm{H}, \mathrm{NH}_{2}\right), 9.58(\mathrm{~s}, 1 \mathrm{H}, \mathrm{CONH}), 1.28-1.93\left(\mathrm{~m}, 10 \mathrm{H}, 5 \mathrm{x} \mathrm{CH}_{2}\right)$, 3.79-3.86 (m, 1H, CH); MS (m/z): $292\left(\mathrm{M}^{+}\right)$; Anal. Calcd for $\mathrm{C}_{15} \mathrm{H}_{24} \mathrm{~N}_{4} \mathrm{O}_{2}$ : C, 61.62; H, 8.27; N, 19.16; Found: C, 61.58; H, 8.15; N, 19.12.

2-Amino- $\mathrm{N}$-(4-fluorophenyl)-4-isopropyl-6-methoxypyrimidine-5-carboxamide(4g):

Yellow solid; mp 180-182 C; IR (KBr): 3459, 3327, 3173, 2989, 1648, 1586, 1261, $1061 \mathrm{~cm}^{-}$ ${ }^{1}$; ${ }^{1} \mathrm{H}$ NMR: $\delta$ 1.19-1.25 (d, 6H, $\left.2 \times{ }^{\mathrm{i}} \mathrm{prCH}_{3}\right), 3.07-3.13\left(\mathrm{~m}, 1 \mathrm{H},{ }_{\mathrm{i}} \mathrm{prCH}\right), 3.82\left(\mathrm{~s}, 3 \mathrm{H}, \mathrm{OCH}_{3}\right)$, $5.69\left(\mathrm{~s}, 2 \mathrm{H}, \mathrm{NH}_{2}\right), 9.58(\mathrm{~s}, 1 \mathrm{H}, \mathrm{CONH}), 7.38-7.40(\mathrm{~d}, 2 \mathrm{H}, \mathrm{Ar}-\mathrm{H}, j=8.8 \mathrm{~Hz})$ 7.63-7.65 (d, 2H, 
Ar-H, $j=8.8 H z)$; MS $(m / z): 304\left(\mathrm{M}^{+}\right)$; Anal. Calcd for $\mathrm{C}_{15} \mathrm{H}_{17} \mathrm{FN}_{4} \mathrm{O}_{2}: \mathrm{C}, 59.20 ; \mathrm{H}, 5.63 ; \mathrm{N}$, 18.41; Found: C, 59.13; H, 5.45; N, 18.52 .

\section{2-Amino- $N$-(4-chlorophenyl)-4-ethoxy-6-isopropylpyrimidine-5-carboxamide (4h):}

white solid; mp 194-196 C; IR (KBr): 3449, 3331, 3182, 3055, 2952, 1651, 1568, 1491, 1247 , $1049 \mathrm{~cm}^{-1}$; ${ }^{1} \mathrm{H}$ NMR: $\delta$ 1.17-1.94 (d, $\left.6 \mathrm{H}, 2 \mathrm{x}{ }_{\mathrm{i}}^{\mathrm{i}} \mathrm{prCH}_{3}\right), 1.28-1.31\left(\mathrm{t}, 3 \mathrm{H}, \mathrm{CH}_{3}\right), 3.05-3.09(\mathrm{~m}$, $\left.1 \mathrm{H},{ }_{1}^{\mathrm{i}} \mathrm{prCH}\right), 4.31-4.36$ (q, $\left.2 \mathrm{H}, \mathrm{CH}_{2}\right), 6.09\left(\mathrm{~s}, 2 \mathrm{H}, \mathrm{NH}_{2}\right), 7.24-7.26(\mathrm{~d}, 2 \mathrm{H}, \mathrm{Ar}-\mathrm{H}, j=8.8 \mathrm{~Hz})$, 7.68-7.70 (d, 2H, Ar-H, j=8.8Hz), $10.07(\mathrm{~s}, 1 \mathrm{H}, \mathrm{CONH}) ; \mathrm{MS}(\mathrm{m} / \mathrm{z}): 334\left(\mathrm{M}^{+}\right)$; Anal. Calcd for $\mathrm{C}_{16} \mathrm{H}_{19} \mathrm{ClN}_{4} \mathrm{O}_{2}$ : C, 57.40; H, 5.72; N, 16.73; Found: C, 57.41; H, 5.55; N, 16.63.

\section{2-Amino- $\mathrm{N}$-(3-chloro-4-fluorophenyl)-4-isopropyl-6-methoxypyrimidine-5-carboxamide}

(4i): Yellow solid; mp 185-187 ${ }^{\circ}$; IR (KBr): 3443, 3325, 3153, 2989, 1648, 1506, 1251, 1064 $\mathrm{cm}^{-1}$; ${ }^{1} \mathrm{H}$ NMR: $\delta$ 1.19-1.25 (d, 6H, $\left.2 \times \mathrm{i}^{\mathrm{i} C H} \mathrm{prCH}_{3}\right), 3.07-3.13(\mathrm{~m}, 1 \mathrm{H}, \mathrm{i}$ prCH, $), 3.82(\mathrm{~s}, 3 \mathrm{H}$, $\left.\mathrm{OCH}_{3}\right), 5.69\left(\mathrm{~s}, 2 \mathrm{H}, \mathrm{NH}_{2}\right), 9.58(\mathrm{~s}, 1 \mathrm{H}, \mathrm{CONH}), 7.80(\mathrm{~s}, 1 \mathrm{H}, \mathrm{Ar}-\mathrm{H}), 7.10-7.12(\mathrm{~d}, 1 \mathrm{H}, \mathrm{Ar}-\mathrm{H}$, $j=8.8 \mathrm{~Hz}), \quad 7.24-7.26,(\mathrm{~d}, 1 \mathrm{H}, \mathrm{Ar}-\mathrm{H}, j=8.8 \mathrm{~Hz})$; $\mathrm{MS}(\mathrm{m} / \mathrm{z})$ : $338\left(\mathrm{M}^{+}\right)$; Anal. Calcd for $\mathrm{C}_{15} \mathrm{H}_{16} \mathrm{ClFN}_{4} \mathrm{O}_{2}$ : C, 53.18; H, 4.76; N, 16.54; Found: C, 53.13; H, 4.65; N, 16.52 .

\section{2-Amino- $N$-(3,4-di-fluorophenyl)-4-isopropyl-6-methoxypyrimidine-5-carboxamide (4j):}

Yellow solid; mp 245-247 C; IR (KBr): 3442, 3226, 3143, 2986, 1642, 1566, 1241, $1061 \mathrm{~cm}^{-1}$; ${ }^{1} \mathrm{H}$ NMR: $\delta 1.19-1.25\left(\mathrm{~d}, 6 \mathrm{H}, 2 \mathrm{x}{ }_{\mathrm{i}}^{\mathrm{i}} \mathrm{prH}_{3}\right), 3.07-3.13\left(\mathrm{~m}, 1 \mathrm{H},{ }_{\mathrm{i}}^{\mathrm{p}} \mathrm{CH},\right), 3.82\left(\mathrm{~s}, 3 \mathrm{H}, \mathrm{OCH}_{3}\right)$, $5.69\left(\mathrm{~s}, 2 \mathrm{H}, \mathrm{NH}_{2}\right), 9.58(\mathrm{~s}, 1 \mathrm{H}, \mathrm{CONH}), 7.90(\mathrm{~s}, 1 \mathrm{H}, \mathrm{Ar}-\mathrm{H}), 7.16-7.18(\mathrm{~d}, 1 \mathrm{H}, \mathrm{Ar}-\mathrm{H}, j=8.8 \mathrm{~Hz})$, 7.26-7.28, (d, 1H,Ar-H, $j=8.8 H z)$; MS $(m / z): 322\left(\mathrm{M}^{+}\right)$; Anal. Calcd for $\mathrm{C}_{15} \mathrm{H}_{16} \mathrm{~F}_{2} \mathrm{~N}_{4} \mathrm{O}_{2}$ : C, 55.90; H, 5.00; N, 17.38; Found: C, 55.73; H, 4.94; N, 17.32.

2-Amino- $N$-(3-chlorophenyl)-4-isopropyl-6-methoxypyrimidine-5-carboxamide $\quad$ (4k): Yellow solid; mp 256-258 ${ }^{\circ}$; IR (KBr): 3459, 3252, 3143, 2919, 1648, 1586, 1241, $1051 \mathrm{~cm}^{-}$ 1. ${ }^{1} \mathrm{H}$ NMR: $\delta 1.19-1.25$ (d, 6H, $\left.2 \times{ }^{\mathrm{i}} \mathrm{prCH}_{3}\right), 3.07-3.13\left(\mathrm{~m}, 1 \mathrm{H},{ }_{\mathrm{i}} \mathrm{prCH},\right), 3.82\left(\mathrm{~s}, 3 \mathrm{H}, \mathrm{OCH}_{3}\right)$, $5.69\left(\mathrm{~s}, 2 \mathrm{H}, \mathrm{NH}_{2}\right), 9.58(\mathrm{~s}, 1 \mathrm{H}, \mathrm{CONH}), 7.92(\mathrm{~s}, 1 \mathrm{H}, \mathrm{Ar}-\mathrm{H}), 7.60-7.62(\mathrm{~d}, 1 \mathrm{H}, \mathrm{Ar}-\mathrm{H}, j=8.8 \mathrm{~Hz})$, 7.51-7.53(d, 1H, Ar-H, $j=8.8 H z), 7.42-7.45(\mathrm{~m}, 1 \mathrm{H}, \operatorname{Ar}-\mathrm{H})$; $\mathrm{MS}(\mathrm{m} / \mathrm{z}): 320\left(\mathrm{M}^{+}\right)$; Anal. Calcd for $\mathrm{C}_{15} \mathrm{H}_{17} \mathrm{ClN}_{4} \mathrm{O}_{2}$ : C, 56.16; H, 5.34; N, 17.47; Found: C, 56.23; H, 5.25; N, 17.52.

\section{2-Amino- $N$-(3,4-di-chlorophenyl)-4-isopropyl-6-methoxypyrimidine-5-carboxamide (4l):}

Yellow solid; mp 240-242 C; IR (KBr): 3420, 3226, 3143, 2988, 1632, 1546, 1231, $1061 \mathrm{~cm}^{-}$ '; ${ }^{1} \mathrm{H}$ NMR: $\delta$ 1.19-1.25 (d, 6H, $\left.2 \times{ }^{\mathrm{i}} \mathrm{prCH}_{3}\right), 3.07-3.10\left(\mathrm{~m}, 1 \mathrm{H},{ }_{\mathrm{i}} \mathrm{prCH},\right), 3.82\left(\mathrm{~s}, 3 \mathrm{H}, \mathrm{OCH}_{3}\right)$, $5.69\left(\mathrm{~s}, 2 \mathrm{H}, \mathrm{NH}_{2}\right), 9.58(\mathrm{~s}, 1 \mathrm{H}, \mathrm{CONH}), 7.93(\mathrm{~s}, 1 \mathrm{H}, \mathrm{Ar}-\mathrm{H}), 7.16-7.18(\mathrm{~d}, 1 \mathrm{H}, \mathrm{Ar}-\mathrm{H}, j=8.8 \mathrm{~Hz})$, 7.28-7.30, $(\mathrm{d}, 1 \mathrm{H}, \mathrm{Ar}-\mathrm{H}, j=8.8 \mathrm{~Hz})$; MS $(\mathrm{m} / z): 354\left(\mathrm{M}^{+}\right)$; Anal. Calcd for $\mathrm{C}_{15} \mathrm{H}_{16} \mathrm{Cl}_{2} \mathrm{~N}_{4} \mathrm{O}_{2}$ : C, 50.72; H, 4.54; N, 15.77; Found: C, 55.73; H, 4.64; N, 15.62.

\section{2-Amino-4-isopropyl-6-methoxy- $N$-p-tolylpyrimidine-5-carboxamide (4m):}

Yellow solid; mp 194-196 C; IR (KBr): 3412, 3317, 3253, 2950, 1613, 1539, 1431, $1061 \mathrm{~cm}^{-}$ 1. ${ }^{1} \mathrm{H}$ NMR: $\delta$ 1.19-1.25 (d, 6H, $\left.2 \times{ }^{\mathrm{i}} \mathrm{prCH}_{3}\right), 3.07-3.13\left(\mathrm{~m}, 4 \mathrm{H},{ }_{\mathrm{i}} \mathrm{prCH},\right), 3.78\left(\mathrm{~s}, 3 \mathrm{H}, \mathrm{OCH}_{3}\right)$, $2.62\left(\mathrm{~s}, 3 \mathrm{H}, \mathrm{CH}_{3}\right), 5.69\left(\mathrm{~s}, 2 \mathrm{H}, \mathrm{NH}_{2}\right), 6.83-6.85(\mathrm{~d}, 1 \mathrm{H}, \mathrm{Ar}-\mathrm{H}, j=8.8 \mathrm{~Hz}), 7.60-7.62(\mathrm{~d}, 1 \mathrm{H}, \mathrm{Ar}-$ $\mathrm{H}, j=8.8 \mathrm{~Hz}), 9.58(\mathrm{~s}, 1 \mathrm{H}, \mathrm{CONH}) ; \mathrm{MS}(\mathrm{m} / z): 300\left(\mathrm{M}^{+}\right)$; Anal. Calcd for $\mathrm{C}_{16} \mathrm{H}_{20} \mathrm{~N}_{4} \mathrm{O}_{2}: \mathrm{C}$, 63.98; H, 6.71; N, 18.65; Found: C, 63.88; H, 6.65; N, 18.52 .

\section{2-Amino-4-isopropyl-6-methoxy- $\mathrm{N}$-o-tolylpyrimidine-5-carboxamide (4n):}

Yellow solid; mp 185-187 C; IR (KBr): 3442, 3327, 3253, 2980, 1623, 1569, 1431, $1051 \mathrm{~cm}^{-}$ 1. ${ }^{1} \mathrm{H}$ NMR: $\delta$ 1.19-1.25 (d, 6H, $\left.2 \times{ }^{\mathrm{i}} \mathrm{prCH}_{3}\right), 3.07-3.13\left(\mathrm{~m}, 1 \mathrm{H},{ }_{\mathrm{i}} \mathrm{prCH},\right), 3.78\left(\mathrm{~s}, 3 \mathrm{H}, \mathrm{OCH}_{3}\right)$, $2.52\left(\mathrm{~s}, 3 \mathrm{H}, \mathrm{Ar}-\mathrm{CH}_{3}\right), 5.69\left(\mathrm{~s}, 2 \mathrm{H}, \mathrm{NH}_{2}\right)$ 7.22-7.24 (d,1H, Ar-H, j=8.8Hz), 7.78-7.80 (d,1H, Ar- 
$\mathrm{H}, j=8.8 H z), 7.51-7.54(\mathrm{~m}, 1 \mathrm{H}, \mathrm{Ar}-\mathrm{H}), 7.57-7.60(\mathrm{~m}, 1 \mathrm{H}, \mathrm{Ar}-\mathrm{H}) \mathrm{MS}(\mathrm{m} / \mathrm{z}): 300\left(\mathrm{M}^{+}\right)$; Anal. Calcd for $\mathrm{C}_{16} \mathrm{H}_{20} \mathrm{~N}_{4} \mathrm{O}_{2}$ : C, 63.98; H, 6.71; N, 18.65; Found: C, 63.88; H, 6.65; N, 18.52 .

2-Amino-4-isopropyl-6-methoxy- $\boldsymbol{N}$-(2-methoxyphenyl)pyrimidine-5-carboxamide (40): Yellow solid; mp 165-167 C; IR (KBr): 3462, 3327, 3220, 2980, 1623, 1509, 1461, $1051 \mathrm{~cm}^{-}$ ; $9.58(\mathrm{~s}, 1 \mathrm{H}, \mathrm{CONH}) ;{ }^{1} \mathrm{H}$ NMR: $\left.\delta 1.19-1.25\left(\mathrm{~d}, 6 \mathrm{H}, 2 \times \mathrm{i}^{\mathrm{i} C H}\right)_{3}\right), 3.07-3.13(\mathrm{~m}, 1 \mathrm{H}$, i $\operatorname{prCH}$ ), $3.78\left(\mathrm{~s}, 3 \mathrm{H}, \mathrm{OCH}_{3}\right), 3.82\left(\mathrm{~s}, 3 \mathrm{H}, \mathrm{OCH}_{3}\right), 5.69\left(\mathrm{~s}, 2 \mathrm{H}, \mathrm{NH}_{2}\right), 3.80\left(\mathrm{~s}, 3 \mathrm{H}, \mathrm{OCH}_{3}\right)$ 7.72-7.74 (d, $1 \mathrm{H}$, Ar-H, $j=8.8 H z), 7.10-7.12(\mathrm{~d}, 1 \mathrm{H}, \mathrm{Ar}-\mathrm{H}, j=8.8 H z), 7.55-7.60(\mathrm{~m}, 1 \mathrm{H}, \mathrm{Ar}-$ $\mathrm{H})$, 7.62-7.64 (m, $1 \mathrm{H}$, Ar-H); MS $(\mathrm{m} / \mathrm{z}): 316\left(\mathrm{M}^{+}\right)$; Anal. Calcd for $\mathrm{C}_{16} \mathrm{H}_{20} \mathrm{~N}_{4} \mathrm{O}_{3}: \mathrm{C}, 60.75$; $\mathrm{H}, 6.37$; N, 17.71; Found: C, 60.68; H, 6.55; N, 17.62 .

\section{2-Amino- $\mathrm{N}$-(2-fluorophenyl)-4-isopropyl-6-methoxypyrimidine-5-carboxamide (4p):}

Yellow solid; mp 178-180 C; IR (KBr): 3442, 3327, 3173, 2989, 1653, 1586, 1261, $1061 \mathrm{~cm}^{-}$ ${ }^{1}$; ${ }^{1} \mathrm{H}$ NMR: $\delta$ 1.19-1.25 (d, 6H, $\left.2 \times{ }^{\mathrm{i}} \mathrm{prCH}_{3}\right), 3.07-3.13\left(\mathrm{~m}, 1 \mathrm{H},{ }_{\mathrm{i}} \mathrm{prCH},\right), 3.82\left(\mathrm{~s}, 3 \mathrm{H}, \mathrm{OCH}_{3}\right)$, $5.69\left(\mathrm{~s}, 2 \mathrm{H}, \mathrm{NH}_{2}\right), 9.58(\mathrm{~s}, 1 \mathrm{H}, \mathrm{CONH}), 7.12-7.14(\mathrm{~d}, 1 \mathrm{H}, \mathrm{Ar}-\mathrm{H}, j=8.8 \mathrm{~Hz}), 7.71-7.73(\mathrm{~d}, 1 \mathrm{H}$ ,Ar-H, $j=8.8 H z), 7.57-7.60(\mathrm{~m}, 1 \mathrm{H}, \operatorname{Ar}-\mathrm{H}), 7.62-7.65(\mathrm{~m}, 1 \mathrm{H}, \operatorname{Ar}-\mathrm{H}) ; \mathrm{MS}(\mathrm{m} / \mathrm{z}): 304\left(\mathrm{M}^{+}\right)$; Anal. Calcd for $\mathrm{C}_{15} \mathrm{H}_{17} \mathrm{FN}_{4} \mathrm{O}_{2}$ : C, 59.20; H, 5.63; N, 18.41; Found: C, 59.16; H, 5.55; N, 18.32 .

\section{2-Amino- $N$-(2-bromophenyl)-4-isopropyl-6-methoxypyrimidine-5-carboxamide (4q):}

Yellow solid; mp 190-192 ${ }^{\circ} \mathrm{C}$; IR (KBr): 3459, 3327, 3173, 2989, 1648, 1586, 1261, $1061 \mathrm{~cm}^{-}$ ' ${ }^{1} \mathrm{H}$ NMR: $\delta$ 1.19-1.25 (d, 6H, $\left.2 \times{ }^{\mathrm{i}} \mathrm{prCH}_{3}\right), 3.07-3.13\left(\mathrm{~m}, 1 \mathrm{H},{ }_{\mathrm{i}} \mathrm{prCH},\right), 3.82\left(\mathrm{~s}, 3 \mathrm{H}, \mathrm{OCH}_{3}\right)$, $5.69\left(\mathrm{~s}, 2 \mathrm{H}, \mathrm{NH}_{2}\right), 9.58(\mathrm{~s}, 1 \mathrm{H}, \mathrm{CONH}), 7.15-7.17(\mathrm{~d}, 1 \mathrm{H}, \mathrm{Ar}-\mathrm{H}, j=8.8 \mathrm{~Hz}), 7.80-7.82(\mathrm{~d}, 1 \mathrm{H}$, $\operatorname{Ar}-\mathrm{H}, j=8.8 H z), 7.55-7.57(\mathrm{~m}, 1 \mathrm{H}, \operatorname{Ar}-\mathrm{H}), 7.60-7.63(\mathrm{~m}, 1 \mathrm{H}, \operatorname{Ar}-\mathrm{H}) ; \mathrm{MS}(\mathrm{m} / \mathrm{z}): 364\left(\mathrm{M}^{+}\right)$; Anal. Calcd for $\mathrm{C}_{15} \mathrm{H}_{17} \mathrm{BrN}_{4} \mathrm{O}_{2}$ : C, 49.33; H, 4.69; N, 15.34; Found: C, 49.13; H, 4.45; N, 15.22 .

\section{2-Amino-4-ethoxy-6-isopropyl- $N$-phenyl pyrimidine-5-carboxamide (4r):}

white solid; $\mathrm{mp} 198-200^{\circ} \mathrm{C}$; IR (KBr): 3459, 3327, 3193, 2999, 1648, 1586, 1261, $1061 \mathrm{~cm}^{-1}$; ${ }^{1} \mathrm{H}$ NMR: $\delta$ 1.19-1.25 (d, 6H, $\left.2 \times \mathrm{i}^{\mathrm{i}} \mathrm{prCH}_{3}\right), 3.07-3.13\left(\mathrm{~m}, 1 \mathrm{H},{ }_{\mathrm{i}} \mathrm{prCH},\right), 5.69\left(\mathrm{~s}, 2 \mathrm{H}, \mathrm{NH}_{2}\right), 9.58$ $(\mathrm{s}, 1 \mathrm{H}, \mathrm{CONH}), 1.29-1.33\left(\mathrm{t}, 3 \mathrm{H}, \mathrm{CH}_{3}\right), 4.32-4.37$ (q, 2H, $\left.\mathrm{CH}_{2}\right), 6.60-6.62(\mathrm{~d}, 2 \mathrm{H}, \mathrm{Ar}-\mathrm{H}$, $j=8.8 H z), 7.10-7.13(\mathrm{~m}, 2 \mathrm{H}, \operatorname{Ar}-\mathrm{H}), 6.40-6.43(\mathrm{~m}, 1 \mathrm{H}, \operatorname{Ar}-\mathrm{H}) ; \mathrm{MS}(\mathrm{m} / \mathrm{z}): 300\left(\mathrm{M}^{+}\right)$; Anal. Calcd for $\mathrm{C}_{16} \mathrm{H}_{20} \mathrm{~N}_{4} \mathrm{O}_{2}$ : C, 63.98; H, 6.71; N, 18.65; Found: C, 63.92; H, 6.65; N, 18.56 .

2-Amino- $\mathrm{N}$-(4-chlorophenyl)-4-isopropyl-6-methoxypyrimidine-5-carboxamide(4s):

Yellow solid; mp 185-187 C; IR (KBr): 3459, 3252, 3143, 2919, 1648, 1586, 1241, $1051 \mathrm{~cm}^{-}$ 1. ${ }^{1} \mathrm{H}$ NMR: $\delta$ 1.19-1.25 (d, 6H, $\left.2 \times{ }^{\mathrm{i}} \mathrm{prCH}_{3}\right), 3.07-3.13\left(\mathrm{~m}, 1 \mathrm{H},{ }_{\mathrm{i}} \mathrm{prCH}\right), 3.82\left(\mathrm{~s}, 3 \mathrm{H}, \mathrm{OCH}_{3}\right)$, $5.69\left(\mathrm{~s}, 2 \mathrm{H}, \mathrm{NH}_{2}\right), 9.58(\mathrm{~s}, 1 \mathrm{H}, \mathrm{CONH}), 7.24-7.26(\mathrm{~d}, 2 \mathrm{H}, \mathrm{Ar}-\mathrm{H}, j=8.8 \mathrm{~Hz}), 7.68-7.70(\mathrm{~d}, 2 \mathrm{H}$, Ar-H, $j=8.8 H z)$; $\mathrm{MS}(\mathrm{m} / \mathrm{z}): 320\left(\mathrm{M}^{+}\right)$; Anal. Calcd for $\mathrm{C}_{15} \mathrm{H}_{17} \mathrm{ClN}_{4} \mathrm{O}_{2}: \mathrm{C}, 56.16 ; \mathrm{H}, 5.34 ; \mathrm{N}$, 17.47; Found: C, 56.20; H, 5.25; N, 17.42 .

\section{2-Amino- $N$-(3-chlorophenyl)-4-ethoxy-6-isopropylpyrimidine-5-carboxamide (4t):}

white solid; $\mathrm{mp} 210-212^{\circ} \mathrm{C}$; IR (KBr): 3449, 3227, 3193, 2966, 1628, 1522, 1217, $1041 \mathrm{~cm}^{-1}$; ${ }^{1} \mathrm{H}$ NMR: $\delta$ 1.19-1.25 (d, 6H, $\left.2 \times{ }^{\mathrm{i}} \mathrm{prCH}_{3}\right), 3.07-3.13\left(\mathrm{~m}, 1 \mathrm{H},{ }_{\mathrm{i}} \mathrm{prCH},\right), 5.69\left(\mathrm{~s}, 2 \mathrm{H}, \mathrm{NH}_{2}\right), 9.58$ (s, 1H, CONH), 1.29-1.33 (t, 3H, $\left.\mathrm{CH}_{3}\right), 4.32-4.37$ (q, 2H, $\left.\mathrm{CH}_{2}\right), 7.92$ (s, 1H, Ar-H), 7.60-7.62 $(\mathrm{d}, 1 \mathrm{H}, \mathrm{Ar}-\mathrm{H}, j=8.8 \mathrm{~Hz}), 7.51-7.53(\mathrm{~d}, 1 \mathrm{H}, \mathrm{Ar}-\mathrm{H}, j=8.8 \mathrm{~Hz}), 7.42-7.45(\mathrm{~m}, 1 \mathrm{H}, \mathrm{Ar}-\mathrm{H}) ; \quad \mathrm{MS}$ $(\mathrm{m} / \mathrm{z})$ : $334\left(\mathrm{M}^{+}\right)$; Anal. Calcd for $\mathrm{C}_{16} \mathrm{H}_{19} \mathrm{ClN}_{4} \mathrm{O}_{2}: \mathrm{C}, 57.40 ; \mathrm{H}, 5.72 ; \mathrm{N}, 16.73$; Found: $\mathrm{C}, 57.44$; $\mathrm{H}, 5.65$; N, 16.67. 


\section{ANTIMICROBIAL SCREENING OF SYNTHESIZED PYRIMIDINES}

All the synthesized compounds were tested against different bacterial and fungal strains i.e. Pseudomonas aeruginosa, Proteus vulgaris, Escherichia Coli, Staphylococcus aureus, Candida albican for their in vitro antibacterial activity. Well Diffusion/Agar Cup Method was used and results are listed in Table 2. The investigation of antibacterial and antifungal screening data revealed that all the tested compounds $4 \mathrm{a}-\mathrm{t}$ showed moderate to potent activity. The compounds $4 \mathrm{i}, 4 \mathrm{k}$ and $4 \mathrm{l}$ showed comparatively good activity against all the bacterial strains.

Table 2. Antibiotic Sensitivity Assay (Concentration 250/500/ $1000 \mu \mathrm{G} / \mathrm{mL}$ ).

\begin{tabular}{|c|c|c|c|c|c|c|c|c|c|c|c|c|c|c|c|}
\hline \multirow[t]{2}{*}{$\begin{array}{c}\text { Code } \\
\text { No. } \\
\end{array}$} & \multicolumn{3}{|c|}{$\begin{array}{c}\text { Pseudomonas } \\
\text { aeruginosa }\end{array}$} & \multicolumn{3}{|c|}{ Proteus vulgaris } & \multicolumn{3}{|c|}{$\begin{array}{c}\text { Escherichia } \\
\text { coli }\end{array}$} & \multicolumn{3}{|c|}{$\begin{array}{c}\text { Staphylococcus } \\
\text { aureus }\end{array}$} & \multicolumn{3}{|c|}{$\begin{array}{l}\text { Candida } \\
\text { albicans }\end{array}$} \\
\hline & 250 & 500 & 1000 & 250 & 500 & 1000 & 250 & 500 & 1000 & 250 & 500 & 1000 & 250 & 500 & 1000 \\
\hline $4 a$ & $\mathrm{R}$ & 1.1 & 1.2 & $\mathrm{R}$ & 1.1 & 1.3 & $\mathrm{R}$ & $\mathrm{R}$ & $\mathrm{R}$ & $\mathrm{R}$ & $\mathrm{R}$ & $\mathrm{R}$ & $\mathrm{R}$ & 1 & 1.2 \\
\hline $4 b$ & 1.2 & 1.4 & 2 & 1.1 & 1.3 & 1.6 & $\mathrm{R}$ & $\mathrm{R}$ & $\mathrm{R}$ & $\mathrm{R}$ & 1 & 1.2 & $\mathrm{R}$ & 1.2 & 1.5 \\
\hline $4 \mathrm{c}$ & 1.2 & 1.3 & 1.7 & 1.1 & 1.4 & 1.6 & $\mathrm{R}$ & $\mathrm{R}$ & $\mathrm{R}$ & 1.2 & 1.3 & 1.6 & 1 & 1.3 & 1.8 \\
\hline $4 \mathrm{~d}$ & 1.1 & 1.3 & 1.5 & $\mathrm{R}$ & 1.1 & 1.4 & 1.1 & 1.2 & 1.3 & $\mathrm{R}$ & 1 & 1.2 & 1.1 & 1.5 & 2 \\
\hline $4 \mathrm{e}$ & 1.1 & 1.2 & 1.4 & 1 & 1.3 & 1.6 & $\mathrm{R}$ & $\mathrm{R}$ & $\mathrm{R}$ & 1.3 & 1.4 & 1.6 & 1.1 & 1.4 & 1.8 \\
\hline $4 \mathrm{f}$ & 1.2 & 1.3 & 1.6 & $\mathrm{R}$ & 1.2 & 1.4 & $\mathrm{R}$ & $\mathrm{R}$ & $\mathrm{R}$ & 1.2 & 1.4 & 1.6 & 1 & 1.3 & 1.7 \\
\hline $4 \mathrm{~g}$ & 1.1 & 1.2 & 1.3 & $\mathrm{R}$ & 1 & 1.2 & $\mathrm{R}$ & $\mathrm{R}$ & $\mathrm{R}$ & 1.2 & 1.3 & 1.5 & 1 & 1.1 & 1.3 \\
\hline $4 \mathrm{~h}$ & 1 & 1.3 & 1.5 & 1.1 & 1.4 & 1.7 & 1.2 & 1.4 & 1.8 & 1.1 & 1.3 & 1.4 & $\mathrm{R}$ & 1.1 & 1.4 \\
\hline $4 \mathrm{i}$ & 1.1 & 1.3 & 1.6 & 1.2 & 1.6 & 2 & 1.3 & 1.5 & 1.9 & 1.1 & 1.5 & 2.2 & 1.2 & 1.6 & 2.3 \\
\hline $4 \mathrm{j}$ & 1.3 & 1.5 & 1.9 & 1 & 1.2 & 1.3 & 1.3 & 1.4 & 1.7 & 1.1 & 1.4 & 1.5 & 1.1 & 1.4 & 1.8 \\
\hline $4 \mathrm{k}$ & 1.3 & 1.5 & 1.8 & 1.1 & 1.4 & 1.7 & 1.2 & 1.4 & 1.8 & 1.4 & 1.5 & 2 & 1.2 & 1.4 & 1.7 \\
\hline 41 & 1.4 & 1.7 & 2 & 1.1 & 1.3 & 1.5 & 1.1 & 1.1 & 1.3 & 1.4 & 1.6 & 2 & 1.1 & 1.3 & 1.5 \\
\hline $4 \mathrm{~m}$ & 1.1 & 1.3 & 1.5 & $\mathrm{R}$ & $\mathrm{R}$ & $\mathrm{R}$ & $\mathrm{R}$ & $\mathrm{R}$ & $\mathrm{R}$ & 1.3 & 1.4 & 1.7 & $\mathrm{R}$ & 1.3 & 1.7 \\
\hline $4 n$ & 1.3 & 1.5 & 1.5 & 1.9 & $\mathrm{R}$ & $\mathrm{R}$ & 1.5 & 1.5 & 1.7 & $\mathrm{R}$ & 1.3 & $\mathrm{R}$ & 1.3 & $\mathrm{R}$ & 1 \\
\hline 40 & 1.5 & 1.6 & 1.3 & 1.1 & 1.4 & 1.3 & 1.4 & 1.7 & 1 & $\mathrm{R}$ & 1.2 & 1.7 & 1.1 & 1.5 & 1.3 \\
\hline $4 p$ & 1.7 & 1.8 & 1.5 & 1 & 1.6 & 1.2 & 1.3 & 1.9 & 1.1 & 1.7 & 1.5 & 1.5 & $\mathrm{R}$ & 1.1 & 1.4 \\
\hline $4 q$ & 1.6 & 1 & 1.2 & 1.5 & 1.4 & 1.2 & 1.2 & 1.5 & 1.4 & 1.6 & 1.8 & 1.3 & 1.5 & 1.3 & 1.8 \\
\hline $4 \mathrm{r}$ & 2 & 1.8 & 1.3 & 1.1 & 1.3 & 1.5 & 1 & 1.2 & 1.5 & 1.1 & 1 & $\begin{array}{l}1.4 \\
\end{array}$ & 1.8 & $\begin{array}{l}1.1 \\
\end{array}$ & 1.6 \\
\hline $4 s$ & 1.2 & 1.1 & 1.1 & 1.7 & 1.8 & 1.4 & 1.1 & 1 & 1.3 & 1.5 & 1.6 & 1.9 & 1.6 & 1 & 2 \\
\hline $4 \mathrm{t}$ & $\mathrm{R}$ & 1 & 2 & 1.3 & 1.3 & 1.2 & 1.5 & 1.7 & 1.2 & 1.3 & 1.2 & 1 & 1.2 & 1.8 & 1.7 \\
\hline $\mathbf{A}$ & \multicolumn{3}{|c|}{1.8} & \multicolumn{3}{|c|}{1.8} & \multicolumn{3}{|c|}{1.9} & \multicolumn{3}{|c|}{1.9} & \multicolumn{3}{|c|}{-} \\
\hline CPD & \multicolumn{3}{|c|}{2.2} & \multicolumn{3}{|c|}{2.1} & \multicolumn{3}{|c|}{2.1} & \multicolumn{3}{|c|}{2.2} & \multicolumn{3}{|c|}{-} \\
\hline GF & \multicolumn{3}{|c|}{1.8} & \multicolumn{3}{|c|}{1.9} & \multicolumn{3}{|c|}{2.0} & \multicolumn{3}{|c|}{2.0} & \multicolumn{3}{|c|}{-} \\
\hline GRF & \multicolumn{3}{|c|}{-} & \multicolumn{3}{|c|}{-} & \multicolumn{3}{|c|}{-} & \multicolumn{3}{|c|}{-} & \multicolumn{3}{|c|}{2.6} \\
\hline FLC & \multicolumn{3}{|c|}{ - } & \multicolumn{3}{|c|}{-} & & - & & & - & & & 2.8 & \\
\hline
\end{tabular}

Note: Zone of inhibition interpretation is as follows.

1. ZONE SIZE <1.0 C.M.- RESISTENT(R)

2. ZONE SIZE 1.0 To 1.5 - INTERMEDIATE

3. ZONE SIZE $>1.5$ - SENSITIVE 


\author{
Std Antibiotic Sensitivity Assay Concentration $40 \mu \mathrm{G} / \mathrm{ml}$ \\ A: Ampicillin \\ CPD: CEFPODOXIME \\ GF: Gatifloxacin \\ GRF: GRESIOFULvin \\ FLC: FLUCONAZOLE
}

\title{
4. CONCLUSION
}

In summary, we have described the synthesis of substituted pyrimidine derivatives in excellent yields by little modification of classical Biginelli reaction. The spectral data are incorporate with the structure of compounds $\mathbf{4 a - j}$. The antimicrobial data reported here which may be the better reference for the future research in the class of pyrimidine derivatives.

\section{Acknowledgement}

Author thankful to Department of Chemistry, Saurashtra University for providing facilities and SAIF, Punjab University for instrumental support.

\section{References}

[1] Undheim K., Benneche T., Gilchrist T. L., Gribble G. W., Advances in Heterocyclic Chemistry; Eds.; Pergamon: Oxford, 11 (1999) 21.

[2] Chan J. H., Hong J. S., Kuyper L. F., Jones M. L., Baccanari D. P., Tansik R. L., Boytos C. M., Rudolph S. K., Brown A. D., J. Heterocycl. Chem. 34 (1997) 145.

[3] Saudi M. N. S., Gaafar M. R., El-Azzouni M. Z., Ibrahim M. A., Eissa M. M., Med. Chem. Res. 17 (2008) 541.

[4] Ren Q., Cui Z., He H., Gu Y., J. Fluorine Chem. 128 (2007) 1369.

[5] Ali A., Taylor G. E., Ellsworth K., Harris G., Painter R., Silver L. L., Young K. J., J. Med. Chem. 46 (2003) 1824.

[6] Tozkoparan B., Ertan M., Kelicen P., Demirdamar R., Il Farmaco 54 (1999) 588.

[7] Biginelli P., Gazz. Chim. Ital. 23 (1893) 360.

[8] Shi F., Jia R., Zhang X., Synthesis 18 (2007) 2782-2790.

[9] Sharma P., Rane N., Gurram V. K., Bioorg. Med. Chem. Lett. 14 (2004) 4185.

[10] Dabiri M., Arvin-Nezhad H., Khavasi H. R., Bazgir A., J. Heterocycl. Chem. 44 (2007) 1009-1011.

[11] Kumar S., Ila H., Junjappa H., Tetrahedron 63 (2007) 10067.

[12] Mahata P., Syam U. K., Sriram V., Ila H., Junjappa H., Tetrahedron 59 (2003) 2631. 